\title{
El educador artístico ante el reto de la inclusión educativa
}

\section{The art educator facing the challenge of educational inclusion}

\author{
Mailyn Gómez* \\ Universidad Central "Marta Abreu" de Las Villas. Santa Clara. Villa Clara. Cuba \\ ORCID: https://orcid.org/0000-0002-4420-7164 \\ Pablo A. Martínez \\ Universidad Central "Marta Abreu" de Las Villas. Santa Clara. Villa Clara. Cuba \\ ORCID: https://orcid.org/0000-0001-5529-8199

\section{Elaine Domínguez} \\ Universidad Central "Marta Abreu" de Las Villas. Santa Clara. Villa Clara. Cuba \\ ORCID: https://orcid.org/0000-0002-9162-864X
}

Received 02-12-20 Revised 06-12-20 Accepted 09-13-20 On line 09-30-20

\section{*Correspondence}

Email:mgsanchez@uclv.cu
Cite as:

Gómez, M., Martínez, P.A., \& Domínguez, E. (2020). El educador artístico ante el reto de la inclusión educativa. Propósitos y Representaciones, 8 (SPE3), e734. Doi: http://dx.doi.org/10.20511/pyr2020.v8nSPE3.734 


\section{Resumen}

El presente trabajo tiene como objetivo fundamentar la necesidad de la preparación de los educadores artísticos que se desempeñan en instituciones educacionales para enfrentar el reto de la inclusión educativa, en la búsqueda de herramientas factibles para su labor, dado el importante papel que desempeñan como promotores de los contenidos de la cultura, se encuentran en una privilegiada posición para propiciar los procesos inclusivos. Se ha podido constatar, a partir de observaciones realizadas en las instituciones educacionales y del análisis de documentos como el plan de estudios y el modelo del profesional de la carrera Licenciatura en Educación. Instructor de arte, que, tanto en la práctica profesional, como en el currículo de la carrera se evidencian limitaciones en cuanto a la preparación de estos especialistas, necesaria para enfrentar el reto de la inclusión educativa en sus contextos de actuación.

Palabras clave: Educador artístico; Inclusión educativa; Necesidades educativas especiales.

\section{Summary}

The purpose of this paper is to base the need for the preparation of artistic educators working in educational institutions to face the challenge of educational inclusion, in the search for feasible tools for their work, given the important role they play as promoters of the contents of the culture, are in a privileged position to promote inclusive processes. It has been possible to verify, based on observations made in educational institutions and the analysis of documents such as the curriculum and the professional model of the degree in Education. Artistic educator, that, both in professional practice and in the curriculum of the career, there are limitations in the preparation of these specialists, necessary to face the challenge of educational inclusion in their contexts of action.

Keywords: Artistic Educator; Educational Inclusion; Special Educational Needs.

\section{Introducción}

La inclusión educativa frecuentemente no es bien comprendida, de ahí la variedad de interpretaciones que pueden encontrarse en la literatura pedagógica y en las prácticas educacionales. En Cuba se concibe a partir del reconocimiento al derecho de todos a una educación de calidad, independientemente de sus particularidades y características que determinan el curso de su desarrollo, que favorezca su integración social como individuos capaces de disfrutar las posibilidades que la sociedad ofrece y al mismo tiempo contribuir a su desarrollo.

Siguiendo estos criterios la inclusión educativa otorga relevancia al alcance de los objetivos socioeducativos por todos los alumnos, no al tipo de institución educacional o modalidad de oferta educativa.

La educación de calidad es aquella que estimula el máximo desarrollo posible en los niños, adolescentes y jóvenes, en consonancia con los resultados deseados y se hace y se justifica cuando es asequible a todos los miembros de la sociedad, cuando facilita los recursos personales, didácticos y metodológicos de que dispone ajustados a las necesidades de cada educando, cuando provoca el cambio en las instituciones educativas, propicia la participación activa de todos los alumnos en su aprendizaje, cuando logra la participación de la familia y los agentes de la comunidad y cuando estimula y facilita el mejoramiento de la preparación de los docentes, especialistas y demás trabajadores (Borges \& Orosco, 2013).

Dentro de los especialistas que se desempeñan en las diferentes instituciones educacionales tienen un importante papel los educadores artísticos, quienes, desde su desempeño como promotores de los contenidos de la cultura, se encuentran en una privilegiada posición para propiciar la inclusión educativa. 
Sin embargo, ante esta tendencia que cobra más fuerza en la Pedagogía y las prácticas educativas en nuestro país, cabe entonces plantear una interrogante: ¿Cuentan los educadores artísticos que desempeñan su labor profesional en las instituciones educativas con la debida preparación para propiciar la inclusión educativa de sus educandos?

En este sentido se ha podido constatar, a partir de observaciones realizadas en las instituciones educacionales y del análisis de documentos como el plan de estudios y el modelo del profesional de la carrera Licenciatura en Educación. Instructor de Arte, que, tanto en la práctica profesional como en el currículo de la carrera se evidencian limitaciones en cuanto a la preparación de estos especialistas, necesaria para enfrentar el reto de la inclusión educativa en sus contextos de actuación.

Precisamente es propósito de los autores de este trabajo, la exposición de sustentos teóricos que permitan fundamentar la necesidad de la preparación de los educadores artísticos que se desempeñan en instituciones educacionales para enfrentar el reto de la inclusión educativa.

\section{Argumentación}

Uno de los principales retos de la práctica educativa actual, teniendo en cuenta el significativo papel que juega el docente como mediador en el proceso educativo, es su preparación como una condición indispensable en la búsqueda de una respuesta verdadera a la diversidad, garantizando al alumno la ayuda necesaria en cada situación, para que todos aprendan más y mejor, potenciando su desarrollo. Al respecto se plantea que existe una estrecha relación entre el reconocimiento de todas las diferencias probables de los alumnos y las posibilidades reales de los docentes para su atención (Bell, 2000).

Como lo menciona Jiménez (2009) se exige del maestro un intenso trabajo de preparación para su desarrollo profesional sobre sólidas bases científicas. La elevación de su nivel de preparación constituye una necesidad, para así poder satisfacer las altas demandas que le plantean nuestro modelo educativo.

En tanto la preparación debe estar dirigida al perfeccionamiento del proceso de enseñanza-aprendizaje, como lo considera Álvarez (2000), un individuo está preparado cuando puede enfrentarse a los problemas que se le presenta y los resuelve.

Es por ello que la preparación del docente, constituye un aspecto muy importante en el desarrollo de los procesos educativos y se concibe como el proceso permanente de adquisición, estructuración y reestructuración de conocimientos, habilidades y valores para el desempeño de su función (Colás, 2012).

Remón \& Escobar (s/f) consideran que:

El docente debe ser capaz de adelantarse a posibles dificultades, desmotivaciones y desaciertos de sus estudiantes tanto en el aprendizaje como en sus aptitudes y conductas, es decir debe poder prever, y en la medida de lo posible evitar situaciones de conflictos y fracasos. El proceso educativo tiene que lograr una integración de influencias, un sistema que opere como una unidad armónica, que fluya sin incoherencia y tenga un carácter sistémico y totalizador, no puede ser una sumatoria de elementos incoherentes. (Remón \& Escobar, s/f, p.2)

En tal sentido se puede afirmar que la preparación constante del docente reclama compromiso, búsqueda y actualización de la información, la integración de conocimientos, exploración en la práctica educativa, esfuerzo por tratar de buscar soluciones y necesidad de superación. 
Ahora, si se reflexiona sobre la preparación de los docentes para una educación inclusiva que respete la diversidad, se puede decir que esta forma de concebir el proceso educativo es un reto para los profesionales de la educación.

La preparación del docente, en este caso, del educador artístico, es decisiva para la puesta en práctica de cualquier proyecto educativo y en tal sentido es necesaria, pues cada estudiante posee estilos y ritmos de aprendizajes diferentes, reconociendo sus necesidades personales, sociales y educativas especiales o no, por lo cual se hace imprescindible la ejecución de un buen diagnóstico como herramienta fundamental que le permita al profesor determinar el estado real del estudiante y su zona de desarrollo próximo; por lo que el profesional de la educación debe estar en constante perfección de sus métodos, técnicas y modos de actuación.

García y Tamayo (2018) consideran que:

Los educadores cubanos debemos continuar perfeccionando desde la teoría lo que hacemos en la práctica social, gestionar nuevos recursos, proyectar mejores alternativas de soluciones a los problemas mediante la introducción y generalización de los resultados de las investigaciones, la sistematización de las experiencias vivenciales y la implementación de políticas educativas inclusivas, como un proceso de oportunidades para todas y todos de acceso al conocimiento (en todas las esferas), de acceso a los servicios (de salud, de seguridad social, cultura), de participación ciudadana, sin distinción por el origen social, sexo, u orientación sexual, color de la piel, lugar de residencia y/o ubicación geográfica, creencias religiosas o nivel profesional. (García \& Tamayo, 2018, p. 5)

Desde el punto de vista sociológico la preparación del educador artístico se sustenta en la concepción de la educación como fenómeno social y contextual, al interactuar con el medio, transformarlo y transformarse a sí mismo en los planos cognitivo, procedimental y actitudinal, para responder a las demandas sociales, lo cual presupone su participación permanente, dirigida a la consolidación de la relación educación-sociedad.

La preparación del educador artístico está sustentada en la idea filosófica de que el hombre es un ser social, históricamente condicionado, producto del desarrollo de la cultura que él mismo crea. El materialismo dialéctico es el método general que ofrece una base teórica esencial para comprender el proceso del conocimiento en el hombre. Particular significación tiene la teoría del conocimiento, la que explica cómo este es el resultado de la interrelación dialéctica del sujeto y los objetos de la realidad, y de los sujetos entre sí, el papel que juega la práctica que en este caso es fundamental, así como la relación entre conocimiento y valoración.

Desde las concepciones filosóficas se asume el trabajo del educador artístico en las instituciones educativas con un enfoque objetivo de los fenómenos éticos y culturales en su acción y desarrollo, desde ella se concretan relaciones internas entre educación, cultura y desarrollo de la personalidad.

El educador artístico es un profesional de la cultura que tiene como encargo social el desarrollo de procesos de promoción, apreciación y creación del arte y de la literatura en la población. Este profesional de la educación se desenvuelve en diferentes contextos de actuación y ejerce una función artístico- pedagógica ya que utiliza las manifestaciones artísticas y literarias en función de la educación de la ciudadanía.

Los educadores artísticos contribuyen con su labor a la formación de valores, actitudes y comportamientos, a través de actividades en diversos contextos de actuación, a partir de las características de cada individuo. Potencia además procesos de apreciación y creación artística desarrollando la imaginación y la personalidad del individuo desde las experiencias adquiridas. 
Estos profesionales en su actuación en las instituciones educativas, según declara la Carta Circular del Ministerio de Educación (MINED) y el Ministerio de Cultura (MINCULT) (2004), deben cumplir los siguientes objetivos:

- el desarrollo de talleres de creación y apreciación con todos los alumnos del centro escolar;

- la atención a grupos y unidades artísticas de aficionados;

- la preparación técnico-metodológica del personal docente;

- la labor promocional de la cultura artística en la escuela;

- el mejoramiento del entorno sonoro y visual de la escuela.

Según dicha resolución además tiene las siguientes funciones:

- Contribuir a la formación integral de los niños, las niñas y los adolescentes acorde a las exigencias de cada grado y ciclo en lo referido a la apreciación de las artes y el desarrollo del gusto estético.

- Trabajar por el rescate, preservación y promoción de la cultura popular y tradicional a partir del respeto a las identidades locales y a la diversidad de sus expresiones y procesos creativos.

- Estimular, promover y desarrollar procesos de apreciación en todas las manifestaciones artísticas.

- Reconocer, valorar y orientar aptitudes y posibilidades para la práctica de todas las manifestaciones artísticas.

- Estimular, promover y desarrollar procesos de creación artística en su especialidad.

- Captar y preparar estudiantes para que en noveno grado opten por la especialidad Educador artístico.

- Utilizar las potencialidades del programa audiovisual y de computación para el desarrollo de la cultura artística.

- Impartir talleres de creación-apreciación en las escuelas de Educación Primaria y Especial, así como talleres de apreciación y de creación en la Secundaria Básica y Especial como parte del currículo.

- Prestar la atención adecuada, según las características del tipo de centro y de sus educandos, a los escolares de la Educación Especial.

- Tener en cuenta para el desarrollo de su labor los objetivos del programa de Educación Estética.

- Propiciar la elevación del gusto y disfrute de la literatura y la mayor utilización del programa Editorial Libertad. 
- Estudiar paulatinamente junto al resto del colectivo pedagógico el modelo de la escuela cubana actual y las características psicológicas de los estudiantes para garantizar efectividad en la labor que desarrollarán.

- Formar, orientar, asesorar y superar al personal docente de la escuela en temas que propicien la elevación de su cultura general e integral, mediante la preparación metodológica y otras vías de trabajo metodológico, así como a otros promotores para el trabajo de su manifestación.

- Diseñar y organizar acciones de trabajo con padres, familiares y otros miembros de la comunidad, para la elevación de su cultura general integral en correspondencia con los diagnósticos.

- Promover la escuela como institución cultural fundamental y su vínculo con las demás instituciones culturales y sociales de la comunidad. Diseñar y organizar las actividades culturales de la escuela en interacción con su comunidad; organizar visitas a las instituciones culturales y sociales, así como la participación de los pioneros de la Primaria y la Secundaria Básica en las actividades que éstas organicen.

- Participar en el diseño e implementación de proyectos comunitarios y en investigaciones socioculturales.

Como bien se puede apreciar, los educadores artísticos tienen la gran responsabilidad de contribuir no solo al logro del vínculo entre la escuela y las instituciones culturales más importantes de la comunidad, abarcando un amplio contexto de actuación, sino también la de prestar atención educativa a los escolares de la Educación Especial.

Sin embargo, estos pedagogos del arte no cuentan con herramientas suficientes para el trabajo en contextos de inclusión educativa, es insuficiente el conocimiento acerca de las necesidades educativas asociadas a discapacidades o no, para darle un tratamiento adecuado a partir de las infinitas potencialidades artísticas con las que cuenta este profesional de la educación.

Si se concibe al arte y la cultura como instrumento pedagógico e ideológico, que posibilita educar e instruir a los pueblos, con la finalidad de alcanzar una vida espiritual plena, socialmente sana, entonces estos profesionales deben ser portadores de un profundo humanismo como principio filosófico que rige la atención a la diversidad.

\section{Según plantea Sánchez (2013):}

Son varias las investigaciones realizadas sobre el papel de las artes en la formación multilateral del hombre, lo que ha servido de base para profundizar y sistematizar la contribución de cada una de las manifestaciones artísticas al desarrollo de las esferas cognoscitiva, afectiva y psicomotora de la personalidad y su influencia en la conducta. De manera similar desde el punto de vista práctico se ha comprobado el efecto de las artes en el sujeto del proceso educativo. (Sánchez, 2013, p. 2)

Los educadores artísticos deben estar preparados para transformar las condiciones en que se desarrolla el proceso educativo si fuese preciso y buscar los métodos, procedimientos y estilos de trabajo que respondan a las particularidades de los grupos y las individualidades de los alumnos que atienden, en función del desarrollo de los mismos. Cada persona puede aprender de manera diferente sobre los mismos objetos.

Las artes adquieren un gran valor que, incluso, las trasciende ontológicamente, pues transforman al individuo en el vínculo constante con las diversas manifestaciones y saberes, se abre a diversos espacios de interacción escolar donde convergen estudiantes, familias y la realidad 
social. Por tanto, se debe tener en cuenta que el trabajo artístico-cultural que desarrolle en los centros educativos se planifique, organice y evalúe en relación con los intereses personales, sociales y comunitarios, desde perspectivas inclusivas, con una proyección integral y desarrolladora.

En las últimas dos décadas surgió una tendencia en los ámbitos nacional e internacional por ampliar y profundizar los derechos humanos, no sólo en cuanto a su alcance, sino también en el impacto social, político y jurídico. Dicha tendencia tiene particular repercusión en la escuela, las prácticas educativas y la Pedagogía como ciencia.

En este sentido Borges (2013) considera que se hace una necesidad inmediata el cambio, la transformación y el desarrollo en aras de brindar una respuesta a las necesidades reales en las instituciones educacionales, para lo cual hace necesario:

La movilización de factores humanos, sociales, culturales, políticos y económicos en función de asegurar a cada persona sin excepción plenas oportunidades y reales posibilidades para su desarrollo, implica una mejor comprensión del fin de la educación de las personas con necesidades educativas especiales, transformar los servicios que se brindan, perfeccionar el trabajo de los equipos multidisciplinarios y operar cambios en la formación del profesorado, perfeccionando el desempeño profesional de los que están en ejercicio. (Borges, 2013, p. 3)

López (2003) al respecto plantea:

La educación debe ser un bien para todos, derecho esencial de las personas, indicador básico de calidad de vida y factor de cohesión, equidad e igualdad de oportunidades, de inclusión social, si se fundamenta en el respeto a las diferencias de cada individuo, si evita la exclusión y pondera la condición de persona por encima de diferencias, limitaciones, ventajas o desventajas. (López, 2003, p.1)

Son varias las investigaciones que se han realizado sobre inclusión educativa, lo que ha permitido que este concepto haya evolucionado hacia la idea de que los estudiantes deben tener oportunidades equitativas de aprendizaje independientemente de su estatus social, cultural, ideológico, físico y de sus diferencias en las habilidades y capacidades.

El origen de la idea de inclusión educativa se sitúa en el foro internacional de la UNESCO que ha marcado pautas en el campo educativo en el evento celebrado en Jomtien, Tailandia (1990), donde se promovió la idea de una Educación para Todos, que ofreciera satisfacción de las necesidades básicas de aprendizaje al tiempo que desarrollara el bienestar individual y social de todas las personas dentro del sistema de educación formal.

Por otra parte, Blanco (2003) considera que la inclusión es un concepto más amplio que el de integración y se relaciona con la naturaleza misma de la educación general y la escuela común. Desde esta perspectiva implica que todos los miembros de una comunidad, más allá de sus condiciones, aprendan juntos.

Otros instrumentos internacionales importantes que han marcado pautas en la tendencia hacia la inclusión educativa se derivan de la Convención de la UNESCO relativa a la lucha contra las discriminaciones en la esfera de la enseñanza (1960), la Convención sobre los Derechos del Niño (1989), instrumento jurídico de derechos humanos más amplio y rápidamente aceptado por la comunidad internacional y la convención sobre los Derechos de las Personas con Discapacidad (2006).

La Conferencia Internacional de 1994, que concluye con la llamada Declaración de Salamanca, marcó una pauta importante, pues se produce una amplia adscripción a la idea de la 
inclusión entre los delegados y se pone énfasis en la urgencia de impartir la enseñanza a todos los niños, jóvenes y adultos, con y sin necesidades educativas especiales dentro un mismo sistema común de educación. La resolución de Salamanca generaliza la inclusión como principio central que ha de guiar la política y la práctica de la construcción de una educación para todos.

En tal sentido la inclusión educativa no está centrada en el tipo de escuela, especial o regular, sino en el alcance de objetivos socioeducativos por todos los educandos, independientemente de la modalidad o institución escolar que brinda la oferta educativa (García \& Tamayo, 2018).

En la Agenda 2030 para el Desarrollo Sostenible el objetivo cuatro Educación de calidad prevé: "Desarrollo de sistemas educativos que fomenten la educación inclusiva de calidad y que promueven las oportunidades de aprendizaje permanente para todos". (UNESCO, 2017, p. 7)

Echeita \& Ainscow (2011), citado por Bros \& Crespo (2019), consideran que, a pesar de existir diferentes definiciones de inclusión, hay cuatro características esenciales que se pueden resaltar, criterio compartido por los autores de este trabajo:

1. La inclusión es un proceso, no es algo que se consiga de la noche a la mañana, sino que requiere unos tiempos y sobre todo constancia. No es una actividad que se hace un día y ya tiene unos resultados, sino que tiene que ser una secuencia de acciones que se van desarrollando progresivamente. Además, hay que tener en cuenta que pueden surgir obstáculos y dificultades con las que hay que lidiar.

2. La inclusión busca la participación de todos los alumnos por igual, sin distinción y con el éxito por igual; es decir, tiene que tener en cuenta a todo el alumnado con sus características propias, considerándolos individuos integrados dentro de un grupo. Para de esta manera poder atender a todos por igual, brindándoles de herramientas suficientes para poder alcanzar exitosamente los objetivos educativos esenciales.

3. La inclusión debe poder identificar barreras y eliminarlas, de forma que si hay alguna actitud que incita al odio o a la exclusión se pueda frenar y evitar.

4. La inclusión se centra en el alumnado en el que puede existir un riesgo de marginación, fracaso escolar o exclusión. Debe prestar especial atención al alumnado que presente estas características, para intervenir a tiempo $\mathrm{y}$, conseguir que adquieran las habilidades educativas exitosamente. (p. 13)

Indudablemente, entender la inclusión educativa desde este punto de vista supone un cambio de paradigma respecto a la ciencia y a las prácticas educativas que se desarrollan en las instituciones educacionales.

En este sentido Almeida \& Castillo (2017) consideran que:

El paradigma de la diversidad supone un cambio profundo de la escuela, que rompa con las antiguas cristalizaciones de la cultura escolar y avance hacia nuevas formas de funcionamiento institucional, todo proceso de cambio en la educación está atravesado por la dimensión política, llevando a revisar las formas en que se inscribe el poder, sus efectos normativos y reproductivos en el funcionamiento institucional. (Almeida \& Castillo, 2017, p.37)

García y Tamayo (2018) defienden que:

La inclusión educativa exige un ajuste en el modelo de escuela actuante, profesores distintos, padres diferentes y una organización escolar de escuela integrada y funcionamiento de comunidad entre todos, que se centre en las habilidades y potencialidades y no en deficiencias y dificultades; 
es preparar a los individuos con y sin necesidades educativas especiales para la vida, siendo sugerente la realización de ajustes en la programación curricular. (García y Tamayo, 2018, p.5)

Por tanto, se puede afirmar que la inclusión obliga a cambios en el proceso pedagógico debiendo los docentes tomar conciencia de este fenómeno social y educacional en aras de una educación de calidad, que respete la diversidad, ajustando planes, programas de estudio, estrategias educativas que atiendan cada necesidad ante las particularidades y dificultades de los estudiantes.

Guirado, García \& Martín (2017) destacan que:

Al ser la diversidad reconocida, como una conducta y condición humana, que se manifiesta en todas las situaciones de la vida cotidiana como actuaciones sociales que expresan el desarrollo personal y la interiorización de esas condiciones sociales, la relación de ambos conceptos permite proyectar los referentes organizadores de la labor docente dirigida a la búsqueda del necesario equilibrio entre la labor educativa del que enseña (docente) y el protagonismo que debe alcanzar el que aprende (escolar), sin embargo, el proceso interactivo no depende sólo de las condiciones personales (de los interactuantes) y del contexto social y cultural en que estos se desarrollan; sino, además, de las acciones y sistemas de acciones de enseñanza y las experiencias de aprendizaje que se proponen a los escolares; así como los aspectos generales que caracterizan cada una de las especialidades de atención a los escolares con necesidades educativas especiales, en las diversas modalidades de atención. (Guirado, García \& Martín, 2017, p. 83)

Actualmente se desarrolla en Cuba el tercer perfeccionamiento educacional, basado en los principios de equidad e inclusión educativa, donde se potencia la preparación de los directores y docentes sobre la base de las exigencias de la ética, la moral y los valores de la profesión, que encuentre su reflejo en una apropiada dirección del trabajo metodológico, además de perfeccionar la adopción de métodos de dirección que promuevan la participación implicada de cada estudiante y de los colectivos de las organizaciones políticas y de masas estudiantiles y pedagógicos en la gestión de su propia educación.

Para el logro de estos propósitos se hace énfasis en el Proyecto Educativo Escolar, concebido como las estrategias que se traza la escuela para dar cumplimiento al fin y los objetivos generales del nivel, dependiendo en gran medida de la capacidad de dirección del centro escolar, integrando todas sus aspiraciones en cuanto a la educación de los estudiantes y las actividades para lograrlas, posibilitando un clima democrático, pues se propone hacerlo con un estilo participativo y articulador, por qué no inclusivo, de todas las potencialidades intraescolares, extraescolares y comunitarias.

Además, se considera que el currículo, atendiendo las exigencias de la sociedad y la atención a la diversidad debe ser integral, flexible y contextualizado, posibilitando la participación activa en la escuela, de sus directivos, profesores, la familia y la comunidad.

En Cuba se igualan las posibilidades reales de conocimiento y oportunidades de desarrollo físico y mental para todos los niños, jóvenes y para todos los ciudadanos en general, sin importar su lugar de residencia, distinción o diferencia de género, edad, color de la piel, credo religioso, político u origen social. Todos los cubanos tienen acceso equitativo a los medios de la educación de manera gratuita, durante todo el proceso de enseñanza.

Cuba asume en el Sistema Nacional de Educación la pedagogía de la diversidad y la inclusión educativa, dirigidas a fortalecer el carácter humanista, socializador, integrador y desarrollador del proceso educativo, independientemente del tipo de institución educacional, de manera que se asegure una educación de calidad, inclusiva, para todos y cada uno de los niños, adolescentes y jóvenes de nuestra sociedad. 
El educador artístico necesita conocer lo que implica la inclusión y debe estar en constante preparación para seguir adquiriendo conocimientos sobre el tema. También debe conocer a cada uno de sus alumnos de manera que pueda adaptar el material que se va trabajar en el aula para poder llegar a todo el alumnado por igual.

\section{Conclusiones}

La inclusión educativa es un proceso que define un conjunto de ideas y prácticas que orientan a la Pedagogía y los procesos educativos en una dirección particular. Los educadores artísticos, en su desempeño en las diferentes instituciones de la educación requieren de la debida preparación en el orden cognitivo, procedimental y actitudinal para asumirla.

El educador artístico como educador en la escuela cubana actual, promotor cultural y agente social importante en la comunidad, tiene como reto importante su continua preparación, la que le permitirá guiar su trabajo en aras del desarrollo educacional y cultural de las nuevas generaciones cumpliendo con su encargo social.

La inclusión de todos los niños, adolescentes y jóvenes en los procesos educativos escolares, mediados por la actuación profesional de todos los docentes de las instituciones educacionales, en particular los educadores artísticos, favorece la apropiación de la cultura, con el correspondiente impacto en el aprendizaje, la formación y desarrollo integral de su personalidad.

\section{Referencias}

Almeida Lozano, G.; Castillo Torres, R. \& Garcés Silva, R. (2017) La percepción del docente ante el reto de la práctica de una educación inclusiva en la unidad educativa "Comandante Rafael Valverde". Ecuador: Universidad de Guayaquil.

Álvarez. C. (2000) "Características esenciales pedagógicas de la escuela cubana"

Bell, R. (2000). Diversidad e integración curricular: implicaciones para la formación docente en Preescolar, Primaria y Especial Educación y diversidad: aristas e implicaciones (pp. 90110). Ciudad de la Habana: Ministerio de Educación.

Borges, S., \& Orosco, M. (2013). Educación especial y educación inclusiva: un horizonte singular y diverso para igualar las oportunidades de desarrollo. La Habana: Sello Editor Educación Cubana.

Bros, S. \& Crespo, T. (2019). "Danza y movimiento como herramienta para la inclusión educativa”. Facultad de Educación y Trabajo social Grado de Educación Infantil. Trabajo fin de Grado Educación Artística

Colás, A. (2012). La formación inicial y permanente del docente: binomio necesario de preparación en la atención a la diversidad Universidad de Ciencias Pedagógicas "Raúl Gómez García".

García Torrell, I. C. \& Tamayo Collado, J. (2018). Las políticas educativas inclusivas en Cuba Educative inclusive politics in Cuba. VARONA, Revista Científico-Metodológica, Edición especial, 5(2), 20-27.

Guirado Rivero, V. C., García Navarro, X., \& Martín González, D. M. (2017). Bases teóricometodológicas para la atención a la diversidad y la inclusión educativa. Universidad y Sociedad, 9(2), 82-88. Recuperado de http://rus.ucf.edu.cu/index.php/rus

Jiménez, C. \& Álvarez, A (2000). Respuesta educativa para la atención a la diversidad.

Llorente, E. (2009). Sistema de actividades teórico-metodológicas para preparar a los docentes para impartir clases de educación musical. (Tesis en Jiménez Opción del Título Académico de Master en Ciencias de la Educación) Instituto Superior Pedagógico "Félix Varela", Sede Municipal Ranchuelo.

Lopez, R. (2003). Igualdad de oportunidades para todos en el sistema educativo. Cuba. 
MINED-MINCULT. (2004). Carta Circular. La Habana. Ministerio de Educación y Ministerio de Cultura de la República de Cuba.

Remón, Y. \& Escobar, A. (s.f.) La atención a la diversidad: un tema necesario en la escuela cubana de hoy. I Congreso online sobre La Educación en el Siglo XXI.

Sánchez Ortega, P. M. (2013). La Educación Artística en Cuba antecedentes y actualidad Atenas, 22(2), 1-51

UNESCO. (2017). La UNESCO Avanza. La Agenda 2030 para el Desarrollo Sostenible. París: Organización de las Naciones Unidas para la Educación, la Ciencia y la Cultura. 